TAIWANESE JOURNAL OF MATHEMATICS

Vol. 5, No. 2, pp. 353-367, June 2001

This paper available online at http://www.math.nthu.edu.tw/tjm/

\title{
NORMAL STRUCTURE AND THE ARC LENGTH IN BANACH SPACES
}

Ji Gao

\begin{abstract}
Let $X$ be a Banach space, $X_{2} \subseteq X$ be a two dimensional subspace of $X$, and $S(X)=\{x \in X,\|x\|=1\}$ be the unit sphere of $X$. The relationship between the normal structure and the arc length in $X$ is studied. Let $R(X)=\inf \left\{l\left(S\left(X_{2}\right)\right)-r\left(X_{2}\right): X_{2} \subseteq X\right\}$, where $l\left(S\left(X_{2}\right)\right)$ is the circumference of $S\left(X_{2}\right)$ and $r\left(X_{2}\right)=\sup \left\{2(\|x+y\|+\|x-y\|): x, y \in S\left(X_{2}\right)\right\}$ is the least upper bound of the perimeters of the inscribed parallelogram of $S\left(X_{2}\right)$. The main result is that $R(X)>0$ implies $X$ has the uniform normal structure.
\end{abstract}

\section{INTRODUCTION}

In a series of papers, Schäffer made use of the concept of geodesic to study the unit sphere of a Banach space $X$ (see [13] for the complete references). He introduced the following two notations: $m(X)=\inf \{\delta(x,-x): x \in S(X)\}$, and $M(X)=\sup \{\delta(x,-x): x \in S(X)\}$ where $S(X)$ is the unit sphere of $X$ and $\delta(x,-x)$ the shortest length of arcs joining antipodal points on $S(X)$. He called $2 \mathrm{~m}(\mathrm{X})$ the girth, and $2 \mathrm{M}(\mathrm{X})$ the perimeter of $X$. These parameters were used to study reflexivity and isomorphism of Banach spaces among other things. But besides $L_{1}$ spaces, $C(K)$ spaces and Hilbert spaces, the values of these parameters are difficult to obtain.

We introduced a geometric parameter $J(X)=\sup \{\|x+y\| \wedge\|x-y\|\}: x, y \in$ $S(X)\}$, a simplification of Schäffer's girth and perimeter, into a Banach space $X$ (see [7] for the complete references). We proved that $J(X)<3 / 2$ implies the uniform normal structure, which, in turn, implies the fixed point property. It is a well-known result that $\delta(1)>0$ implies normal structure, where $\delta(\epsilon)$ is the modulus

Received February 2, 2000; revised August 29, 2000.

Communicated by P. Y. Wu.

2000 Mathematics Subject Classification: 46B20.

Key words and phrases: Arc length, modulus of convexity, normal structure, uniformly nonsquare space, uniform normal structure and ultraproduct space. 
of convexity. We gave an example of a Banach space $X$ with $J(X)<3 / 2$ and $\delta(1)=0$ to show the significance of the parameter $J(X)$. We also computed the values of $J(X)$ for some classical Banach spaces (see Appendix, §6), and posted a related question as to whether uniformly nonsquare Banach spaces have the fixed point property.

In this paper, we introduce another geometric parameter, $R(X)$, into a Banach space $X$, and prove that for a Banach space $X, R(X)>0$ implies the uniform normal structure. We then give in $\S 4$ an example of a Banach space $X$ with $R(X)>0$ and $J(X)>3 / 2$. Significantly, this means that the parameter $R(X)$ is really distinct from $J(X)$. However, whether uniformly nonsquare Banach spaces have the fixed point property is still an open question.

\section{PRELIMINARIES}

Let $\mathrm{X}$ be a normed linear space, and let $S(X)=\{x \in X:\|x\|=1\}$ be the unit sphere of $X$.

\section{2-1. Curves in Banach Spaces}

A continuous mapping $x(t)$ from a closed interval $[a, b]$ to a Banach space $X$ is called a curve in $X: C=x(t), a \leq t \leq b$. A curve is called simple if it does not have multiple points. A curve is called closed if $x(a)=x(b)$. A closed curve is called symmetric about the origin if $x \in C$, then also $-x \in C$.

The concept of the length of a curve in Banach spaces resembles the same concept in Euclidean spaces. For curve $C=x(t)$, let $\mathrm{P}$ stand for a partition $a=t_{0}<t_{1}<t_{2}<\ldots<t_{i}<\ldots<t_{n}=b$ of interval $[a, b]$ and $l(C, P)=$ $\sum_{i=1}^{n}\left\|x\left(t_{i}\right)-x\left(t_{i-1}\right)\right\|$, where $x_{i}(t), i=0,1,2, \ldots, n$ are called partition points on $C$. Then the length $l(C)$ of curve $C=x(t), a \leq t \leq b$, is defined as the least upper bound of $l(C, P)$ for all possible partitions $P$ of $[a, b]$ :

$$
l(C)=\sup _{P}\{l(C, P)\} .
$$

If $l(C)$ is finite, the curve is called rectifiable.

Let $\|P\|=\max _{1 \leq i \leq n}\left\{\left|t_{i}-t_{i-1}\right|\right\}$ for a partition $P$ of $[a, b]$.

Theorem $1[2,13]$. If curve $C$ is rectifiable, then for all $\epsilon>0$, there exists $\delta>0$ such that $\|P\|<\delta$ implies $l(C)-l(C, P)<\epsilon$. Furthermore, if $\left\{P_{k}\right\}$ is a sequence of partitions of $[a, b]$ with $\left\|P_{k}\right\| \rightarrow 0$, then $\lim _{k \rightarrow \infty} l\left(C, P_{k}\right)=l(C)$.

Let $l_{a}^{t}(C)$ denote the length of curve $C=x(t)$ from $a$ to $t$. For a rectifiable curve $C=x(t), a \leq t \leq b$, the arc length $l_{a}^{t}(C)$ is a continuous function of $t$. 
Definition $1[2,13]$. Let $y(s)$ represent the point $x(t)$ on the curve $C$ for which $l_{a}^{t}(C)=s$. Then $C=y(s), 0 \leq s \leq l(C)$, is called the standard form of the rectifiable curve $C$.

For a normed linear space $\mathrm{X}$, we use $X_{2}$ to denote a two-dimensional subspace of X. Then $S\left(X_{2}\right)$ is a simple closed curve which is symmetric about the origin and unique up to orientation.

Theorem 2 [2,13]. Let $X_{2}$ be a two-dimensional Banach space, and $K_{1}, K_{2}$ be closed convex subsets of $X_{2}$ with nonvoid interiors. If $K_{1} \subseteq K_{2}$, then $l\left(\partial\left(K_{1}\right)\right) \leq$ $l\left(\partial\left(K_{2}\right)\right)$, where $l\left(\partial\left(K_{i}\right)\right)$ denotes the length of the circumference of $K_{i}, i=1,2$.

Theorem 3 [13]. $l\left(S\left(X_{2}\right)\right) \leq 8 ; l\left(S\left(X_{2}\right)\right)=8$ if and only if $S\left(X_{2}\right)$ is a parallelogram.

Theorem 4 [13]. $l\left(S\left(X_{2}\right)\right) \geq 6 ; l\left(S\left(X_{2}\right)\right)=6$ if and only if $S\left(X_{2}\right)$ is an affinely regular hexagon.

\section{2-2. Normal Structure in Banach Spaces}

In 1948, Brodskii and Milman [1] introduced the following geometric concepts:

Definition 2. A bounded, convex subset $K$ of a Banach space $X$ is said to have normal structure if every convex subset $H$ of $K$ that contains more than one point contains a point $x_{0} \in H$ such that $\sup \left\{\left\|x_{0}-y\right\|, y \in H\right\}<d(H)$, where $d(H)=\sup \{\|x-y\|, x, y \in H\}$ denotes the diameter of $H$. A Banach space $X$ is said to have normal structure if every bounded, convex subset of $X$ has normal structure. A Banach space $X$ is said to have weak normal structure if for each weakly compact convex set $K$ in $X$ that contains more than one point has normal structure. $X$ is said to have uniform normal structure if there exists $c, 0<c<1$, such that for any subset $K$ as above, there exists $x_{0} \in K$ such that $\sup \left\{\left\|x_{0}-y\right\|\right.$, $y \in K\}<c \cdot(d(K))$.

For a reflexive Banach space $\mathrm{X}$, the normal structure and weak normal structure coincide.

In 1964, Kirk [10] proved that if a weakly compact subset $K$ of $X$ has normal structure then any nonexpansive mapping on $K$ has a fixed point. Since then much attention has been focused on normal structure. Whether or not a Banach space has normal structure depends on the geometry of the unit sphere. We refer the interested reader to $[4,5,6,7,8,11,15,16]$. 
Lemma 1 [5]. Let $X$ be a Banach space without weak normal structure. Then for any $\epsilon, 0<\epsilon<1$, there exists a sequence $\left\{z_{n}\right\} \subseteq S(X)$ with $z_{n} \rightarrow^{w} 0$, and

$$
1-\epsilon<\left\|z_{n+1}-z\right\|<1+\epsilon
$$

for sufficiently large $n$ and any $z \in \operatorname{co}\left\{z_{k}\right\}_{k=1}^{n}$.

Lemma 2 [7]. Let $X$ be a Banach space without weak normal structure. Then for any $\epsilon, 0<\epsilon<1$, there exist $x_{1}, x_{2}, x_{3}$ in $S(X)$ satisfying

(i ) $x_{2}-x_{3}=a x_{1}$ with $|a-1|<\epsilon$,

(ii) ||$\left|x_{1}-x_{2}\right||-1|,||\left|x_{3}-\left(-x_{1}\right)\right||-1|<\epsilon$, and

(iii) $\left\|\left(x_{1}+x_{2}\right) / 2\right\|,\left\|\left(x_{3}-x_{1}\right) / 2\right\|>1-\epsilon$.

The geometric meaning of the lemma can be succinctly described as follows: if $X$ does not have weak normal structure, then there exists an inscribed hexagon in $S(X)$ with length of each side arbitrarily closed to 1 (by (i) and (ii)), and with at least four sides whose distance to $S(X)$ are arbitrarily small (by (iii)).

\section{Parameter $R(X)$ and Normal Structure}

For a Banach space $X$, let $B(X)=\{x \in X:\|x\| \leq 1\}$ be the ball of $X$, $B_{0}(X)=B(X) \backslash S(X)$ be the interior of $B(X)$. If $K \subseteq X$, let $\operatorname{co}(K)$ be the convex hull of subset $K$ of $X$.

If $x, y \in S\left(X_{2}\right)$, then $2(\|x+y\|+\|x-y\|)$ is the perimeter of inscribed parallelogram with vertices $x, y,-x$, and $-y$ of $S\left(X_{2}\right)$.

Let $r\left(X_{2}\right)=\sup \left\{2(\|x+y\|+\|x-y\|): x, y \in S\left(X_{2}\right)\right\}$. Then $r\left(X_{2}\right) \leq$ $l\left(S\left(X_{2}\right)\right)$ by Theorem 2 .

Definition 3. For a Banach space $X$, define $R(X)=\inf \left\{l\left(S\left(X_{2}\right)\right)-r\left(X_{2}\right)\right.$ : $\left.X_{2} \subseteq X\right\}$.

For a Hilbert space $H, R(H)=2 \pi-4 \sqrt{2}$.

Theorem 5. If $X$ is a Banach space with $R(X)>0$, then $X$ is uniformly nonsquare.

Proof. Suppose $X$ is not uniformly nonsquare. For any $\epsilon>0$, there exist $x, y \in S(X)$ such that both $\|x+y\|$ and $\|x-y\|>2-(\epsilon / 4)$ [9]. let $X_{2}$ be the twodimensional space spanned by $x$ and $y$. Then $r\left(X_{2}\right) \geq 2(\|x+y\|+\|x-y\|)>8-\epsilon$, and hence $R(X)=\inf \left\{l\left(S\left(X_{2}\right)\right)-r\left(X_{2}\right): X_{2} \subseteq X\right\}<\epsilon$. Since $\epsilon$ can be arbitrarily small, we have $R(X)=0$. 
Lemma 3 [7]. Let $x, y \in B(X)$ and $0<\epsilon<1$ such that $\|x+y\| / 2>1-\epsilon$. Then for any $c, 0 \leq c \leq 1$, and $z=c x+(1-c) y \in \operatorname{co}(\{x, y\})$, the line segment connecting $x$ and $y$, we have $\|z\|>1-2 \epsilon$.

Theorem 6. If $X$ is a Banach space with $R(X)>0$, then $X$ has normal structure.

Proof. $R(X)>0$ implies that $\mathrm{X}$ is uniformly nonsquare, and hence reflexive [9]. Normal structure and weak normal structure coincide.

Suppose $X$ dose not have weak normal structure. For $\epsilon>0$, let $x_{1}, x_{2}$ and $x_{3}$ in $\mathrm{S}(\mathrm{X})$ satisfy the conditions in Lemma 2 . Let $X_{2}$ be the two-dimensional space spanned by $x_{1}$ and $x_{2}$ (see the Figure below).

Then there exists an $x \in X$ and a real number $\alpha$ such that $x-x_{2}=\alpha\left(x_{2}-x_{1}\right)$ and $x-x_{3}=\alpha\left(x_{3}+x_{1}\right)$. Let $y_{2} \in$ the line segment $c o\left(\left\{x, x_{2}\right\}\right)$ and $y_{3} \in$ the line segment $c o\left(\left\{x, x_{3}\right\}\right)$, and $\beta$ be the real number such that $y_{2}-x_{2}=\beta\left(x_{2}-x_{1}\right), y_{3}-$ $x_{3}=\beta\left(x_{3}+x_{1}\right), c o\left(\left\{y_{2}, y_{3}\right\}\right) \bigcap S(X) \neq \varnothing$, and $c o\left(\left\{y_{2}, y_{3}\right\}\right) \subseteq X_{2} \backslash B_{0}\left(X_{2}\right)$. Then $y_{2}-y_{3}=\delta\left(x_{2}-x_{3}\right)$, where $0<\delta<1$. Furthermore, we can take $\epsilon$ small enough such that $\beta<(1+\epsilon) /(1-\epsilon)<2$. Then

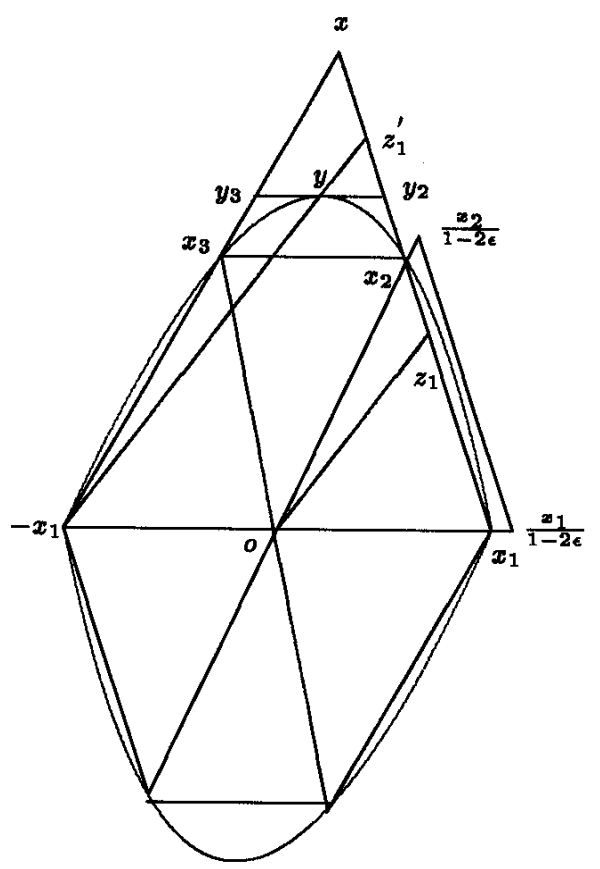

Figure 


$$
\begin{gathered}
\left\|y_{2}-x_{2}\right\|=\beta\left\|x_{2}-x_{1}\right\| \leq \beta(1+\epsilon) \leq \beta+2 \epsilon, \\
\left\|y_{3}-x_{3}\right\|=\beta\left\|x_{3}+x_{1}\right\| \leq \beta(1+\epsilon) \leq \beta+2 \epsilon, \\
\frac{\left\|x_{2}-x_{1}\right\|}{\left\|x-x_{2}\right\|}=\frac{2-\left\|x_{2}-x_{3}\right\|}{\left\|x_{2}-x_{3}\right\|} \geq \frac{1-\epsilon}{1+\epsilon} \geq 1-2 \epsilon, \\
\delta=\frac{\left\|y_{2}-y_{3}\right\|}{\left\|x_{2}-x_{3}\right\|}=\frac{\left\|x-y_{2}\right\|}{\left\|x-x_{2}\right\|}=1-\frac{\left\|y_{2}-x_{2}\right\|}{\left\|x-x_{2}\right\|} \\
=1-\frac{\left\|y_{2}-x_{2}\right\|}{\left\|x_{2}-x_{1}\right\|} \cdot \frac{\left\|x_{2}-x_{1}\right\|}{\left\|x-x_{2}\right\|} \leq 1-\beta(1-2 \epsilon) \leq 1-\beta+4 \epsilon,
\end{gathered}
$$

and

$\left\|y_{2}-y_{3}\right\|=\delta\left\|x_{2}-x_{3}\right\| \leq(1-\beta+4 \epsilon)(1+\epsilon) \leq 1-\beta+4 \epsilon+2 \epsilon=1-\beta+6 \epsilon$.

Therefore, the length of the curve from $x_{2}$ to $x_{3}$ on $S\left(X_{2}\right)$ is less than or equal to $2(\beta+2 \epsilon)+1-\beta+6 \epsilon=1+\beta+10 \epsilon$.

Since $\left\|x_{1}+x_{2}\right\| / 2>1-\epsilon$, for any $z \in \operatorname{co}\left(\left\{x_{1}, x_{2}\right\}\right)$, we have $\|z\|>1-2 \epsilon$. So, the line segment $\operatorname{co}\left(\left\{x_{1} /(1-2 \epsilon), x_{2} /(1-2 \epsilon)\right\}\right) \subseteq X_{2} \backslash B_{0}\left(X_{2}\right)$, and hence the length of the curve from $x_{1}$ to $x_{2}$ on $S\left(X_{2}\right) \leq$ the sum of the lengths of the line segments $\operatorname{co}\left(\left\{x_{1}, x_{1} /(1-2 \epsilon)\right\}\right)$, and $\operatorname{co}\left(\left\{x_{1} /(1-2 \epsilon), x_{2} /(1-2 \epsilon)\right\}\right)$, and $\operatorname{co}\left(\left\{x_{2} /(1-2 \epsilon), x_{2}\right\}\right) \leq 2 /(1-2 \epsilon)-2+(1+\epsilon) /(1-2 \epsilon) \leq 1+8 \epsilon$.

Similarly, the length of the curve from $x_{3}$ to $-x_{1}$ on $S\left(X_{2}\right)$ is less than or equal to $1+8 \epsilon$.

Therefore, $l\left(S\left(X_{2}\right)\right)=2$ (length of the curve from $x_{1}$ to $x_{2}$ on $S\left(X_{2}\right)+$ length of the curve from $x_{2}$ to $x_{3}$ on $S\left(X_{2}\right)+$ length of the curve from $x_{3}$ to $-x_{1}$ on $S\left(X_{2}\right) \leq 2(2(1+8 \epsilon)+1+\beta+10 \epsilon)=2(3+\beta+26 \epsilon)=6+2 \beta+52 \epsilon$. We have

$$
l\left(S\left(X_{2}\right)\right) \leq 6+2 \beta+52 \epsilon .
$$

On the other hand, let $y \in \operatorname{co}\left(\left\{y_{2}, y_{3}\right\}\right) \cap S\left(X_{2}\right)$. There must exist an $z_{1}^{\prime} \in$ $\operatorname{co}\left(\left\{x_{2}, x\right\}\right)$ and $z_{1} \in \operatorname{co}\left(\left\{x_{1}, x\right\}\right)$ such that $y \in \operatorname{co}\left(\left\{-x_{1}, z_{1}^{\prime}\right\}\right)$, and $\left\|z_{1}^{\prime}+x_{1}\right\|=$ $2\left\|z_{1}\right\|$. If $z_{1} \in \operatorname{co}\left(\left\{x_{1}, x_{2}\right\}\right)$, then $\left\|z_{1}\right\| \geq 1-2 \epsilon$, and hence $\left\|z_{1}^{\prime}+x_{1}\right\|=2\left\|z_{1}\right\| \geq$ $2(2-2 \epsilon)=2-4 \epsilon$. Since $\left\|z_{1}^{\prime}-y\right\| /\left\|z_{1}\right\|=\left\|y-y_{2}\right\| /\left\|x_{1}\right\|,\left\|z_{1}^{\prime}-y\right\|=\left\|z_{1}\right\|(\| y-$ $\left.y_{2} \|\right) \leq\left\|y-y_{2}\right\|$, and $\left\|y+x_{1}\right\|=\left\|z_{1}^{\prime}+x_{1}\right\|-\left\|z_{1}^{\prime}-y\right\| \geq 2-4 \epsilon-\left\|y-y_{2}\right\|$. If $z_{1} \in \operatorname{co}\left(\left\{x_{2}, x\right\}\right)$, then $\left\|z_{1}^{\prime}+x_{1}\right\|=2\left\|z_{1}\right\| \geq 2$. We need the following fact.

Fact: Suppose $u=x_{2}+t\left(x_{2}-x_{1}\right), u_{1}=x_{2}+t_{1}\left(x_{2}-x_{1}\right), t_{1} \geq 0$, and $\left\|u_{1}+x_{1}\right\| \geq 2$. Then $\left\|u+x_{1}\right\|$ is an increasing function of $t$ on $\left[t_{1}, \infty\right)$.

Proof of the fact: Let $U(x, a)=\{y \in X:\|y-x\| \leq a\}$ and $S(x, a)=\{y \in$ $X:\|y-x\|=a\}$ be the unit ball and the unit sphere of $X$ with center at $x$ and 
radius $a$, respectively. Since $\left\|x_{2}-\left(-x_{1}\right)\right\| \leq 2$, there exists $v_{1} \in \operatorname{co}\left(\left\{x_{2}, u_{1}\right\}\right)$ such that $v_{1} \in S\left(-x_{1}, 2\right)$.

If $\left\|u+x_{1}\right\|$ is not an increasing function, let $t_{1} \leq t_{2} \leq t_{3}$ such that $\left\|u_{2}+x_{1}\right\|=$ $b>\left\|u_{3}+x_{1}\right\|$, where $u_{2}=x_{2}+t_{2}\left(x_{2}-x_{1}\right)$, and $u_{3}=x_{2}+t_{3}\left(x_{2}-x_{1}\right)$. Since $v_{1} \in \operatorname{co}\left(\left\{x_{2}, u_{1}\right\}\right) \subseteq \operatorname{co}\left(\left\{x_{2}, u_{2}\right\}\right), b \geq 2$ by the convexity of $U\left(-x_{1}, 2\right)$.

Consider $v_{2}=2\left(u_{2}+x_{1}\right) / b-x_{1}$, and $v_{3}=2\left(u_{3}+x_{1}\right) / b-x_{1}$. Then $v_{2} \in$ $S\left(-x_{1}, 2\right)$, and $v_{3} \in U\left(-x_{1}, 2\right) \backslash S\left(-x_{1}, 2\right)$. Since $u_{2}=c v_{1}+(1-c) u_{3}$, where $0 \leq c \leq 1$, we have $u_{2}+x_{1}=c\left(v_{1}+x_{1}\right)+(1-c)\left(u_{3}+x_{1}\right),\left\|u_{2}+x_{1}\right\| \leq$ $c\left\|v_{1}+x_{1}\right\|+(1-c)\left(\left\|u_{3}+x_{1}\right\|\right)$, and $b\left\|v_{2}+x_{1}\right\| / 2 \leq 2 c+b(1-c)\left\|v_{3}+x_{1}\right\| / 2$. Therefore $\left\|v_{2}+x_{1}\right\|<2(2 c+(1-c) b) / b \leq 2$. This contradicts with $v_{2} \in S\left(-x_{1}, 2\right)$.

From the previous fact we have $\left\|z_{1}^{\prime}+x_{1}\right\| \leq\left\|x+x_{1}\right\| \leq 2(1+\epsilon) /(1-\epsilon) \leq 2+6 \epsilon$. Hence $\left\|z_{1}\right\| \leq 1+3 \epsilon$, and $\left\|z_{1}^{\prime}-y\right\|=\left\|z_{1}\right\|\left(\left\|y-y_{2}\right\|\right) \leq(1+3 \epsilon)\left(\left\|y-y_{2}\right\|\right)$. So, $\left\|y+x_{1}\right\|=\left\|z_{1}^{\prime}+x_{1}\right\|-\left\|z_{1}^{\prime}-y\right\| \geq 2-(1+3 \epsilon)\left\|y-y_{2}\right\| \geq 2-4 \epsilon-\left\|y-y_{2}\right\|$. Finally, we proved $\left\|y+x_{1}\right\| \geq 2-4 \epsilon-\left\|y-y_{2}\right\|$.

Similarly, $\left\|y-x_{1}\right\| \geq 2-4 \epsilon-\left\|y-y_{3}\right\|$.

So, $\left\|y+x_{1}\right\|+\left\|y-x_{1}\right\| \geq 4-8 \epsilon-\left(\left\|y-y_{2}\right\|+\left\|y-y_{3}\right\|\right)=4-8 \epsilon-\left\|y_{2}-y_{3}\right\| \geq$ $4-8 \epsilon-(1-\beta+6 \epsilon)=3+\beta-14 \epsilon$, and hence

$$
r\left(X_{2}\right)=\sup \left\{2(\|x+y\|+\|x-y\|): x, y \in S\left(X_{2}\right)\right\} \geq 6+2 \beta-28 \epsilon .
$$

From (3.1) and (3.2), we have $R(X)=\inf \left\{l\left(S\left(X_{2}\right)\right)-r\left(X_{2}\right): X_{2} \subseteq X\right\}<80 \epsilon$. Since $\epsilon$ can be arbitrarily small, we have $R(X)=0$.

\section{4. $\mathrm{R}(\mathrm{X})$ AND Other Parameters}

Let $\delta(\epsilon)=\inf \{1-(\|x+y\| / 2):\|x-y\| \geq \epsilon, x, y \in S(X)\}, 0 \leq \epsilon \leq 2$, be the modulus of convexity of $X$. Since $\inf \{1-(\|x+y\|) / 2:\|x-y\| \geq \epsilon, x, y \in$ $S(X)\}=\inf \{1-(\|x+y\|) / 2:\|x-y\|=\epsilon, x, y \in S(X)\}, 0 \leq \epsilon \leq 2$, we have $\delta(\|x-y\|) \leq 1-(\|x+y\|) / 2$, for any $x, y \in S(X)$.

Let $l(X)=\inf \left\{l\left(S\left(X_{2}\right)\right): X_{2} \subseteq X\right\}$. Then $6 \leq l(X) \leq 8$.

Lemma 4. For a Banach space $X, \delta\left(2^{-}\right)>0$, where $\delta\left(2^{-}\right)=\lim _{\epsilon \rightarrow 2} \delta(\epsilon)$, implies that $X$ is uniformly nonsquare.

Proof. If $X$ is not uniformly nonsquare, let $x, y \in S(X)$ be as in Theorem 5 . Then $\delta\left(2-(\epsilon / 4) \leq 1-(2-(\epsilon / 4)) / 2=\epsilon / 8\right.$. Letting $\epsilon \rightarrow 0$, we have $\delta\left(2^{-}\right)=0$. 
Theorem 7. For a Banach space $X, \delta(l(X) / 4)>2-(l(X) / 4$ implies $X$ has normal structure.

Proof. $\delta(l(X) / 4)>2-(l(X) / 4)$ implies $\delta\left(2^{-}\right)>0$, so $X$ is uniformly nonsquare. Hence $X$ is reflexive, therefore weak normal structure and normal structure coincide.

If $X$ fails to have normal structure, for any $\epsilon>0$, let $x_{1}, x_{2}, x_{3}$ and $y$ be in Theorem 6. Then $1-\epsilon \leq\left\|y-x_{1}\right\| \leq 2,1-\epsilon \leq\left\|y+x_{1}\right\| \leq 2$, and $l(X) / 2-80 \epsilon \leq$ $\left\|y-x_{1}\right\|+\left\|y+x_{1}\right\|$, from Theorem 6. So, $l(X) / 2-2-80 \epsilon \leq \min \left\{\left\|y-x_{1}\right\|\right.$, $\left.\left\|y+x_{1}\right\|\right\}$, and $\max \left\{\left\|y-x_{1}\right\|,\left\|y+x_{1}\right\|\right\} \geq l(X) / 4-40 \epsilon$. Recall that $\delta(\epsilon)$ is an increasing function on $[0,2]$. Thus $\delta(l(X) / 4-40 \epsilon) \leq \delta\left(\max \left\{\left\|y-x_{1}\right\|\right.\right.$, $\left.\left.\left\|y+x_{1}\right\|\right\}\right) \leq 1-\min \left\{\left\|y-x_{1}\right\|,\left\|y+x_{1}\right\|\right\} / 2 \leq 2-l(X) / 4+40 \epsilon$. By letting $\epsilon \rightarrow 0$, we have $\delta(l(X) / 4) \leq 2-l(X) / 4$.

Therefore, $\delta(l(X) / 4)>2-l(X) / 4$ implies normal structure.

Corollary 1. For a Banach space $X$ with $l(X) \geq 7$, the condition $\delta(7 / 4)>$ $1 / 4$ implies $X$ has normal structure.

Proof. The conditions $l(X) / 4 \geq 7 / 4,2-l(X) / 4 \leq 1 / 4, \delta(7 / 4)>1 / 4$, and $\delta(\epsilon)$ is an increasing function on $[0,2]$ imply that $\delta(l(X) / 4)>2-l(X) / 4$. So, $X$ has normal structure from Theorem 7 .

Since $\delta(3 / 2)>1 / 4$ implies $\delta(7 / 4)>1 / 4$, Corollary 1 improved the result of Corollary 5.6 for the space $X$ with $l(X) \geq 7$ [7].

Corollary 2. For a Banach space $X$, if there exists an $\epsilon$, such that $0 \leq \epsilon \leq$ $l(X) / 4$ and $\delta(\epsilon)>((8-l(X)) / l(X)) \epsilon$, then $X$ has normal structure.

Proof. $\delta(\epsilon) / \epsilon$ is an increasing function on [0, 2] by [12]. $\delta(\epsilon) / \epsilon>(8-$ $l(X)) / l(X)(0 \leq \epsilon \leq l(X) / 4)$ implies $\delta(l(X) / 4) /(l(X) / 4) \geq \delta(\epsilon) / \epsilon>(8-$ $l(X)) / l(X)$, that is, $\delta(l(X) / 4)>2-l(X) / 4$.

Theorem 8. For a Banach space $X, \delta\left(2^{-}\right)>1 / 2$ implies $X$ has normal structure.

Proof. If $X$ fails to have normal structure, let $x_{1}, x_{2}, x_{3}$ be in Lemma 2. Then $2-4 \epsilon \leq\left\|x_{3}-x_{1}\right\| \leq 2,1-\epsilon \leq\left\|x_{3}+x_{1}\right\| \leq 1+\epsilon$, and $\delta(2-4 \epsilon) \leq \delta\left(\left\|x_{3}-x_{1}\right\|\right) \leq$ $1-\left(\left\|x_{3}+x_{1}\right\| / 2\right) \leq(1+\epsilon) / 2$. By letting $\epsilon \rightarrow 0$, we have $\delta\left(2^{-}\right) \leq 1 / 2$.

Corollary 3. For a Banach space $X$, the condition $\delta(\epsilon)>\epsilon / 4$ implies $X$ has normal structure.

Proof. Since $\delta(\epsilon) / \epsilon$ is an increasing function on [0, 2], from $\delta\left(2^{-}\right) / 2 \geq$ $\delta(\epsilon) / \epsilon>1 / 4$, we have $\delta\left(2^{-}\right)>1 / 2$.

Let $r(X)=\sup \left\{r\left(X_{2}\right): X_{2} \subseteq X\right\}=\sup \{2(\|x+y\|+\|x-y\|): x, y \in$ $S(X)\}$. 
Proposition 1. If $X$ is a Banach space, either $l_{p}$ or $L_{p}[0,1]$, then $r(X)=$ $2^{2+(1 / p)}, 1<p \leq 2 ; r(X)=2^{2+(1 / q)}, p>2$, where $(1 / p)+(1 / q)=1$.

Proof. By using Lagrange multipliers in basic calculus, the function $u+v$, under the constraint $u^{p}+v^{p}=a$, assumes its maximum $2^{(p-1) / p} \cdot a^{1 / p}$ at $u=v=(a / 2)^{1 / p}$.

If $1<p \leq 2$, Clarkson inequality [3, 4]: $\|x+y\|^{p}+\|x-y\|^{p} \leq 2\left(\|x\|^{p}+\|y\|^{p}\right)$, for all $x, y \in S(X)$, implies that $\|x+y\|+\|x-y\| \leq 2^{(p-1) / p} \cdot 2^{2 / p}=2^{(p+1) / p}$.

If $p>2$, Clarkson inequality $\|x+y\|^{p}+\|x-y\|^{p} \leq 2^{p-1}\left(\|x\|^{p}+\|y\|^{p}\right)$, for all $x, y \in S(X)$, implies $\|x+y\|+\|x-y\| \leq 2^{(p-1) / p} \cdot\left(2^{p}\right)^{1 / p}=2^{(2 p-1) / p}$.

For $l_{p}, 1<p \leq 2$, let $x=(1,0,0, \ldots, 0, \ldots)$ and $y=(0,1,0, \ldots, 0, \ldots)$. Then $\|x+y\|+\|x-y\|=2^{(p+1) / p}$.

For $L_{p}[0,1], 1<p \leq 2$, let

$$
x(t)= \begin{cases}2^{\frac{1}{p}}, & 0 \leq t<\frac{1}{2} \\ 0, & \frac{1}{2} \leq t \leq 1\end{cases}
$$

and

$$
y(t)= \begin{cases}0, & 0 \leq t<\frac{1}{2} \\ 2^{1 / p}, & \frac{1}{2} \leq t \leq 1\end{cases}
$$

Then

$$
\|x(t)+y(t)\|+\|x(t)-y(t)\|=\sqrt[p]{\int_{0}^{1}\left(2^{\frac{1}{p}}\right)^{p} d t}+\sqrt[p]{\int_{0}^{1}\left(2^{\frac{1}{p}}\right)^{p} d t}=2 \cdot 2^{\frac{1}{p}}=2^{\frac{p+1}{p}} .
$$

We have $r(X)=\sup \{2(\|x+y\|+\|x-y\|): x, y \in S(X)\}=2^{(1 / p)+2}$, $1<p \leq 2$.

For $l_{p}, p>2$, let $x=\left(2^{-1 / p}, 2^{-1 / p}, 0, \ldots, 0, \ldots\right), y=\left(2^{-1 / p},-2^{-1 / p}, 0, \ldots, 0, \ldots\right)$. Then $\|x+y\|+\|x-y\|=2\left(2^{(p-1) / p}\right)=2^{(p+1) / p}$.

For $L_{p}[0,1], p>2$, let

$$
x(t)= \begin{cases}1, & 0 \leq t<\frac{1}{2} \\ 1, & \frac{1}{2} \leq t \leq 1\end{cases}
$$

and

$$
y(t)= \begin{cases}-1, & 0 \leq t<\frac{1}{2} \\ 1, & \frac{1}{2} \leq t \leq 1\end{cases}
$$

Then

$$
\|x(t)+y(t)\|+\|x(t)-y(t)\|=\sqrt[p]{\int_{\frac{1}{2}}^{1} 2^{p} d t}+\sqrt[p]{\int_{0}^{\frac{1}{2}} 2^{p} d t}=2\left(2^{\frac{p-1}{p}}\right)=2^{\frac{2 p-1}{p}} .
$$


We have $r(X)=\sup \{2(\|x+y\|+\|x-y\|): x, y \in S(X)\}=2^{3-(1 / p)}=$ $2^{2+(1 / q)}, p>2$.

Theorem 9. For a Banach space $X, r(X)<l(X)$ implies $X$ has normal structure.

Proof. $R(X)=\inf \left\{l\left(S\left(X_{2}\right)\right)-r\left(X_{2}\right): X_{2} \subseteq X\right\} \geq \inf \left\{l\left(S\left(X_{2}\right)\right): X_{2} \subseteq\right.$ $X\}-\sup \left\{r\left(X_{2}\right): X_{2} \subseteq X\right\}=l(X)-r(X) . r(X)<l(X)$ implies $R(X)=$ $l(X)-r(X)>0$, which hence implies $X$ has normal structure by Theorem 6 .

Corollary 4. For a Banach space $X, r(X)<6$ implies that $X$ has normal structure.

Finally, at the end of this section we show that the two parameters are distinct by giving an example of a Banach space $X$ with $R(X)>0$ and $J(X)>3 / 2$.

Consider an $n$-dimensional space $l_{p}^{n}$, where $1 \leq p \leq \infty$, and $n$ is a positive integer. The norm is defined by

$$
\left\|\left(x_{1}, x_{2}, \ldots, x_{n}\right)\right\|_{p}= \begin{cases}\left(\sum_{j=1}^{n}\left|x_{j}\right|^{p}\right)^{\frac{1}{p}}, & \text { if } 1 \leq p<\infty \\ \max \left\{\left|x_{1}\right|,\left|x_{2}\right|, \ldots,\left|x_{n}\right|\right\}, & \text { if } p=\infty\end{cases}
$$

This is a subspace of general $l_{p}$ space, where $1 \leq p \leq \infty$. The norm is defined by

$$
\left\|\left(x_{1}, x_{2}, \ldots, x_{n}, \ldots\right)\right\|_{p}= \begin{cases}\left(\Sigma_{j=1}^{\infty}\left(\left|x_{j}\right|^{p}\right)^{\frac{1}{p}},\right. & \text { if } 1 \leq p<\infty \\ \sup \left\{\left|x_{1}\right|,\left|x_{2}\right|, \ldots,\left|x_{n}\right|, \ldots\right\}, & \text { if } p=\infty .\end{cases}
$$

From [7],

$$
J\left(l_{p}\right)= \begin{cases}2^{1-\frac{1}{p}}, & \text { if } 2 \leq p<\infty \\ 2, & \text { if } p=\infty .\end{cases}
$$

It is easy to show $J\left(l_{p}^{n}\right)=J\left(l_{p}\right)$ for all $n$.

Let $n=2$, and $p>\left(\log _{2}(4 / 3)\right)^{-1}$. Then $J\left(l_{p}^{2}\right)=2^{1-(1 / p)}>2 \cdot 3 / 4=3 / 2$.

On the other hand, $S\left(l_{p}^{2}\right)$ is a compact set in $\mathbb{R}^{2}$, so there exist $x$ and $y \in S\left(l_{p}^{2}\right)$ such that the supremum is assummed at $x$ and $y$ in the definition of $J\left(l_{p}^{2}\right)$. So, $J\left(l_{p}^{2}\right)=\|x+y\|=\|x-y\|$, and $r\left(l_{p}^{2}\right)=2(\|x+y\|+\|x-y\|)=4\|x+y\|$.

But $l_{p}^{2}$ is a two-dimensional uniform convex space, so $l\left(S\left(l_{p}^{2}\right)\right)>4\|x+y\|$ by the definition of arc length. We have $R\left(l_{p}^{2}\right)>0$.

We may also use an $l_{p}^{n}$ space for any $n$ or the $l_{p}$ space to establish our purpose, but for the $l_{p}$ space it is more complicated. 


\section{The Arc Lengths and Uniform Normal Structure}

Let $F$ be a filter on an index set $I$, and let $\left\{x_{i}\right\}_{i \in I}$ be a subset in a Hausdorff topological space $X .\left\{x_{i}\right\}_{i \in I}$ is said to converge to $x$ with respect to $F$, denoted by $\lim _{F} x_{i}=x$, if for each neighborhood $U$ of $x,\left\{i \in I: x_{i} \in U\right\} \in F$. A filter $U$ on $I$ is called an ultrafilter if it is maximal with respect to the ordering of the set inclusion. An ultrafilter is called trivial if it is of the form $\left\{A: A \subseteq I, i_{0} \in A\right\}$ for some $i_{0} \in I$. We will use the fact that if $U$ is an ultrafilter, then (i): for any $A \subseteq I$, either $A \subseteq U$ or $I \backslash A \subseteq U$; (ii): if $\left\{x_{i}\right\}_{i \in I}$ has a cluster point $x$, then $\lim _{U} x_{i}$ exists and equals $x$.

Let $\left\{X_{i}\right\}_{i \in I}$ be a family of Banach spaces and let $l_{\infty}\left(I, X_{i}\right)$ denote the subspace of the product space equipped with the norm $\left\|\left(x_{i}\right)\right\|=\sup _{i \in I}\left\|x_{i}\right\|<\infty$.

Definition 4 [14]. Let $U$ be an ultrafilter on $I$ and let $N_{U}=\left\{\left(x_{i}\right) \in l_{\infty}\left(I, X_{i}\right)\right.$ : $\left.\lim _{U}\left\|x_{i}\right\|=0\right\}$.

The ultraproduct of $\left\{X_{i}\right\}_{i \in I}$ is the quotient space $l_{\infty}\left(I, X_{i}\right) / N_{U}$ equipped with the quotient norm.

We will use $\left(x_{i}\right)_{U}$ to denote the element of the ultraproduct. It follows from remark (ii) above and the definition of quotient norm that

$$
\left\|\left(x_{i}\right)_{U}\right\|=\lim _{U}\left\|x_{i}\right\| .
$$

In the following we will restrict our index set $I$ to be $\mathbb{N}$, the set of natural numbers, and let $X_{i}=X, i \in \mathbb{N}$, for some Banach space $X$. For an ultrafilter $U$ on $\mathbb{N}$, we use $X_{U}$ to denote the ultraproduct.

Theorem 10. For any Banach space $X$, and for any nontrivial ultrafilter $U$ on $\mathbb{N}, R\left(X_{U}\right)=R(X)$.

Proof. For any $\epsilon>0$, from the definition of $R(X)$, there exists a twodimensional subspace $X_{2} \subseteq X$ and $x, y \in S\left(X_{2}\right)$ such that for all partitions $P$ of the interval $\left[0, l\left(S\left(X_{2}\right)\right)\right]$ and the corresponding $l\left(S\left(X_{2}\right), P\right)$,

$$
l\left(S\left(X_{2}\right), P\right)-2(\|x+y\|+\|x-y\|)<R(X)+\epsilon .
$$

Let $x_{i}=x$, and $y_{i}=y$, for all $i \in \mathbb{N}$. Then $\left(x_{i}\right)_{U},\left(y_{i}\right)_{U} \in S\left(\left(X_{U}\right)_{2}\right)_{\text {, }}$ where $\left(X_{U}\right)_{2}$ is a two dimensional subspace, spanned by $\left(x_{i}\right)_{U}$, and $\left(y_{i}\right)_{U}$, of $X_{U}$. The projection from $X_{U}$ to $X$ produces a one-to-one correspondence between the partitions $P_{U}$ of $\left[0, l\left(S\left(\left(X_{U}\right)_{2}\right)\right)\right]$ and the partition $P$ of $\left[0, l\left(S\left(X_{2}\right)\right)\right]$, and $l\left(S\left(\left(X_{U}\right)_{2}\right), P_{U}\right)=l\left(S\left(X_{2}\right), P\right)$.

Hence $l\left(S\left(\left(X_{U}\right)_{2}\right), P_{U}\right)-2\left(\left\|\left(x_{i}\right)_{U}+\left(y_{i}\right)_{U}\right\|+\left\|\left(x_{i}\right)_{U}-\left(y_{i}\right)_{U}\right\|\right)=l\left(S\left(X_{2}\right), P\right)-$ $2(\|x+y\|+\|x-y\|)<R(X)+\epsilon$.

Since $\epsilon$ can be arbitrarily small, we have proved $R\left(X_{U}\right) \leq R(X)$. 
To prove the reverse inequality, we choose $\left(X_{U}\right)_{2} \subseteq X_{U},\left(x_{i}\right)_{U},\left(y_{i}\right)_{U} \in$ $S\left(\left(X_{U}\right)_{2}\right)$, and a partition $P_{U}$ of $\left[0, l\left(S\left(\left(X_{U}\right)_{2}\right)\right)\right]$ such that $l\left(S\left(\left(X_{U}\right)_{2}\right), P_{U}\right)>$ $l\left(S\left(\left(X_{U}\right)_{2}\right)\right)-\epsilon$ and $l\left(S\left(\left(X_{U}\right)_{2}\right), P_{U}\right)-2\left(\left\|\left(x_{i}\right)_{U}+\left(y_{i}\right)_{U}\right\|+\left\|\left(x_{i}\right)_{U}-\left(y_{i}\right)_{U}\right\|\right)<$ $R\left(X_{U}\right)+\epsilon$.

Without loss of generality, we may assume that $\left\|x_{i}\right\|,\left\|y_{i}\right\|=1$ for all $i \in \mathbb{N}$, and the norm of each component of the partition on $S\left(\left(X_{U}\right)_{2}\right)$ has norm 1 too.

From Theorem 1 and (5.1), $l\left(S\left(\left(X_{U}\right)_{2}\right)\right)=\sup _{P_{U}}\left\{l\left(S\left(\left(X_{U}\right)_{2}\right), P_{U}\right)\right\}$ $=\sup _{P_{U}}\left\{\lim _{U}\left\{l\left(S\left(X_{2}^{i}\right),\left(P_{U}\right)_{i}\right)\right\}\right\}=\lim _{U}\left\{\sup _{P_{U}}\left\{l\left(S\left(X_{2}^{i}\right),\left(P_{U}\right)_{i}\right)\right\}\right\}$

$=\lim _{U}\left\{l\left(S\left(X_{2}^{i}\right)\right)\right\}$, where $X_{2}^{i}$ is a two-dimensional subspace spanned by $x_{i}$, and $y_{i}$, and $\left(P_{U}\right)_{i}$, a projection of the partition $P_{U}$ to $X_{2}^{i}$, is a partition of $S\left(X_{2}^{i}\right)$ for all $i \in \mathbb{N}$.

From remarks (i) and (ii) of ultrafilter and by (5.1) and the paragragh above, the sets

$J=\left\{i \in \mathbb{N}, l\left(S\left(\left(X_{U}\right)_{2}\right), P_{U}\right)-2\left(\left\|\left(x_{i}\right)_{U}+\left(y_{i}\right)_{U}\right\|+\left\|\left(x_{i}\right)_{U}-\left(y_{i}\right)_{U}\right\|\right)<R\left(X_{U}\right)+\epsilon\right\}$,

$K=\left\{i \in \mathbb{N}, l\left(S\left(\left(X_{U}\right)_{2}\right), P_{U}\right)>l\left(S\left(\left(X_{U}\right)_{2}\right)\right)-\epsilon\right\}$, and

$M=\left\{i \in \mathbb{N}, l\left(S\left(X_{2}^{i}\right)\right)<l\left(S\left(\left(X_{U}\right)_{2}\right)\right)+\epsilon\right\}$

are all in $U$. So the intersection $J \bigcap K \bigcap M$ is in $U$ too, and is hence not empty.

Let $i \in J \bigcap K \bigcap M$. We have $l\left(S\left(X_{2}^{i}\right),\left(P_{U}\right)_{i}\right)-2\left(\left\|x_{i}+y_{i}\right\|+\left\|x_{i}-y_{i}\right\|\right)<$ $R\left(X_{U}\right)+\epsilon, l\left(S\left(X_{2}^{i}\right),\left(P_{U}\right)_{i}\right)>l\left(S\left(\left(X_{U}\right)_{2}\right)\right)-\epsilon$, and $l\left(S\left(X_{2}^{i}\right)\right)<l\left(S\left(\left(X_{U}\right)_{2}\right)\right)+\epsilon$.

So, $l\left(S\left(X_{2}^{i}\right)\right)-2\left(\left\|x_{i}+y_{i}\right\|+\left\|x_{i}-y_{i}\right\|\right)<R\left(X_{U}\right)+3 \epsilon$. Hence $R(X)<$ $R\left(X_{U}\right)+3 \epsilon$. Since $\epsilon$ can be arbitrarily small, $R(X) \leq R\left(X_{U}\right)$.

Similarly, we can prove the following two theorems:

Theorem 11. For any Banach space $X$, and for any nontrivial ultrufilter $U$ on $\mathbb{N}, r\left(X_{U}\right)=r(X)$.

Theorem 12. For any Banach space $X$, and for any nontrivial ultrufilter $U$ on $\mathbb{N}, l\left(X_{U}\right)=l(X)$.

Theorem 13. If $X$ is a Banach space with $R(X)>0$, then $X$ has uniform normal structure.

Proof. The idea of the proof is the same as the proof of Theorem 4.4 in [7]. Suppose that $R(X)>0$, and that $X$ does not have uniform normal structure. We find a sequence $\left\{C_{n}\right\}$ of bounded closed convex subsets of $X$ such that for each $n$,

$0 \in C_{n}, d\left(C_{n}\right)=1$, and

$\operatorname{rad}\left(C_{n}\right)=\inf \left\{\sup \left\{\|x-y\|, y \in C_{n}\right\}, x \in C_{n}\right\}>1-\frac{1}{n}$.

Let $U$ be any nontrivial ultrafilter on $\mathbb{N}$, and let

$$
C=\left\{\left(x_{n}\right)_{U}: x_{n} \in C_{n}, n \in \mathbb{N}\right\} .
$$


Then $C$ is a nonempty bounded closed convex subset of $X_{U}$. It follows from the above properties of $C_{n}$ that $d(C)=\operatorname{rad}(C)=1$, so $X_{U}$ does not have normal structure. On the other hand, from Theorem 10, $R\left(X_{U}\right)=R(X)>0$. This contradicts Theorem 6 , and hence $X$ must have uniform normal structure.

Similarly, we can prove the following theorem:

Theorem 14. For a Banach space $X, \delta(l(X) / 4)>2-(l(X) / 4)$ implies that $X$ has uniform normal structure.

Ttheorem 15. For a Banach space $X, r(X)<l(X)$ implies that $X$ has uniform normal structure.

Theorem 16. For a Banach space $X, r(X)<6$ implies that $X$ has uniform normal structure.

\section{APPENDIX}

In this section, I summarize some results about the parameters $\delta(\epsilon), r(X)$ and $J(X)$ for some classical Banach spaces.

Theorem 17 [7]. Let $X$ be either $l_{p}$ or $L_{p}[0,1]$, where $1 \leq p \leq \infty$. Then $J(X)=2^{1 / p}$, if $1<p \leq 2 ; J(X)=2^{1-(1 / p)}$, if $2<p<\infty$; and $J(X)=2$, if $p=1$ or $\infty$.

Theorem $18\left[4\right.$, p. 148]. Let $X$ be either $l_{p}$ or $L_{p}[0,1]$, where $1<p<\infty$. Then, $\delta(\epsilon)$ satisfies the equation: $(1-\delta(\epsilon)+(\epsilon / 2))^{p}+(1-\delta(\epsilon)-(\epsilon / 2))^{p}=2$, if $1<p \leq 2 ; \delta(\epsilon)=1-\left(1-(\epsilon / 2)^{p}\right)^{1 / p}$, if $2<p<\infty$.

Theorem 19. For the spaces $l_{1}, l_{\infty}, L_{1}[0,1]$ and $L_{\infty}[0,1]$, we have $\delta(\epsilon) \equiv 0$.

Proof. From [7], for any Banach space $X, J(X)<\epsilon$ if and only if $\delta(\epsilon)>$ $1-(\epsilon / 2)$. So, it is a direct result of Theorem 17.

The values of $r(X)$ are shown in Proposition 1.

\section{ACKNOWLEDGEMENTS}

The author is very grateful to the referee for several constructive suggestions and improvements. 


\section{REFERENCES}

1. M. S. Brodskii and D. P. Milman, On the center of a convex Set, Dokl. Akad. Nauk $\operatorname{SSSR}(N . S$.) $\mathbf{5 9}$ (1948), 837-840.

2. H. Buseman, The Geometry of Geodesics, Academic Press, New York, 1955.

3. J. A. Clarkson, Uniformly convex spaces, Trans. Amer. Math. Soc. 40 (1936), 396-414.

4. M. M. Day, Normed Linear Spaces, 2nd ed., Springer-Verlag, New York, 1973.

5. D. van Dulst, Some more Banach spaces with normal structure, J. Math. Anal. Appl. 104 (1984), 285-289.

6. D. van Dulst and B. Sims, Fixed points of nonexpansive mappings and Chebyshev centers in Banach spaces with norms of type (KK), in: Banach Space Theory and Its Applications (Bucharest, 1981) Lecture Notes in Mathematics 991, Springer-Verlag, 1983.

7. J. Gao and K. S. Lau, On two classes of Banach spaces with normal structure, Studia Math. 99 (1991), 41-56.

8. R. Huff, Banach spaces which are nearly uniformly convex, Rocky Mountain J. Math. 10 (1980), 743-749.

9. R. C. James, Uniformly nonsquare Banach spaces, Ann. Math. 80 (1964), 542-550.

10. W. A. Kirk, A fixed point theorem for mappings which do not increase distance, Amer. Math. Monthly 72 (1965), 1004-1006.

11. T. Landes, Normal structure and the sum-property, Pacific J. Math. 123 (1986), 127-147.

12. J. Lindenstrauss and L. Tzafriri, Classical Banach Spaces II, Function Spaces, Springer-Verlag, Berlin, 1979.

13. J. J. Schäffer, Geometry of Spheres in Normed Spaces, Marcel Dekker, New York, 1976.

14. B. Sims, "Ultra"-Techniques in Banach Spaces Theory, Queen's papers in Pure and Appl. Math., 60, Queen's University, Kingston, Ontario, 1982.

15. B. Turett, A dual view of a theorem of Baillon, in: Nonlinear Analysis and Applications, Lecture Notes in Pure and Appl. Math., 80, Marcel Dekker, New York, 1982, pp. 279-286.

16. V. Zizler, On some rotundity and smoothness properties of Banach spaces, Dissertationes Math. (Rozprawy Mat). 87 (1971), 5-33.

Department of Mathematics

Community College of Philadelphia

Philadelphia, PA 19130-3991, USA

E-mail: jgao@ccp.cc.pa.us 